\title{
Análise de Fatores Associados à Ulceração de Extremidades em Indivíduos Diabéticos com Neuropatia Periférica
}

artigo original

\author{
MARIANA V.P. PORCIÚNCULA \\ LUIz Clemente P. Rolim \\ LUCIANA GAROFOLO \\ SANDRA ROBERTA G. FERREIRA
}

Programa de Pós-Graduação em Ciências Endocrinológicas (MVPP \& LCPR) e Programa de Pós-Graduação em Cirurgia

Vascular (LG), Universidade

Federal de São Paulo; e Departamento de Nutrição da Faculdade de Saúde Pública (SRGF), Universidade de São Paulo, São Paulo, SP.

Recebido em 01/03/07 Aceito em 18/06/07

\section{RESUMO}

A neuropatia periférica é o principal fator de risco para ulceração em pé de indivíduos diabéticos. Este estudo testou a associação de doença arterial periférica (DAP) à ulceração do pé em amostra de pacientes com neuropatia sensório-motora simétrica distal e se marcadores inflamatórios subclínicos também se associariam a esse evento. Foram avaliados 32 indivíduos diabéticos tipo $2 \mathrm{com}$ exame do monofilamento de $10 \mathrm{~g}$ alterado, estratificados em 2 grupos segundo a história ou presença de úlcera nas extremidades inferiores. O grupo "com úlcera" $(n=18)$ incluiu aqueles que apresentavam úlcera ativa ou cicatrizada, ou que tiveram alguma amputação em membro inferior decorrente de complicações da úlcera. Além do exame neurológico e monofilamento, foram submetidos a bioestesiometria, avaliação vascular com Doppler e exames laboratoriais. Os grupos foram semelhantes quanto à distribuição dos sexos, média de idade e tempo de diabetes. O grupo com úlcera apresentou valores médios de altura $(1,70 \pm 0,06$ vs. 1,63 $\pm 0,11 \mathrm{~m} ; \mathrm{p}=$ $0,044)$ e limiar de percepção vibratória no maléolo medial $(40,9 \pm 13,0$ vs. $30,6 \pm 12,3 \mathrm{~V} ; \mathrm{p}=$ 0,040 ) mais elevados que o sem a úlcera. Os grupos não diferiram entre si quanto à média dos marcadores inflamatórios. A resposta do reflexo patelar foi também pior no grupo com úlcera $(p=0,047)$, no qual se observou maior proporção de indivíduos com o índice háluxbraquial alterado $(p=0,030)$ quando comparado ao sem úlcera. Conclui-se que a DAP está associada à presença de úlcera (atual ou pregressa) em membros inferiores de indivíduos diabéticos neuropatas. A pesquisa de alteração de fluxo de artérias digitais de membro inferior (no hálux) contribuiu para detectar tal associação. Associação de neuropatia ulcerada a marcadores inflamatórios não foi observada, não sendo possível excluí-la devido às limitações do tamanho da amostra. Estudos prospectivos deverão examinar a sensibilidade do índice hálux-braquial para identificar DAP em indivíduos diabéticos sob risco de ulceração. (Arq Bras Endocrinol Metab 2007;51/7:1134-1142)

Descritores: Neuropatia diabética periférica; Úlcera de pé; Doença arterial periférica; Índice hálux-braquial; Marcadores inflamatórios

\section{ABSTRACT}

Analysis of Factors Associated with Extremity Ulceration in Diabetic Subjects with Peripheral Neuropathy.

Peripheral neuropathy is the main risk factor for foot ulceration in diabetic subjects. This study examined the association of peripheral arterial disease (PAD) with foot ulceration in a sample of diabetic subjects with peripheral neuropathy, and also if inflammatory markers would be associated with this event. We evaluated 32 type 2 diabetic individuals with abnormal 10-g monofilament exam, who were stratified in 2 groups according to history or presence of lower extremities ulcer. The group "with ulcer" $(n=18)$ included the ones that had active or cicatrized ulcer, or some lower-extremity amputation due to ulcer complications. In addition to the neurological examination and monofilament test, they were submitted to biothesiometry, lower extremity vascular assessment with Doppler, and laboratory determinations. No difference between the groups was found concerning sex distribution, mean age, and duration of diabetes diagnosis. The group with ulcer showed higher mean values of height $(1.70 \pm 0.06$ vs. $1.63 \pm 0.11 \mathrm{~m}, \mathrm{p}=0.044)$, vibration perception threshold measured in medial malleolli $(40.9 \pm 13.0$ vs. $30.6 \pm 12.3 \mathrm{~V}, \mathrm{p}=0.040)$ than the group without ulcer. The groups did not differ regarding the mean values of the inflammatory markers. Response to patellae reflex was worse in the group with ulcer $(p=0.047)$, in which a higher proportion of individuals with abnormal toe-brachial index $(p=0.030)$ was observed as compared to those without ulcer. We concluded that PAD is associated with the presence of ulcer in neuropathic subjects. The assessment of digital arteries flow in lower limbs (in great toe) contributed to detect such association. Association of diabetic foot ulcers and inflammatory markers was not observed, but cannot be excluded due to limitations of sample size. Prospective studies should examine the sensitivity of the toe-brachial index to identify PAD in diabetic individual at risk of ulceration. (Arq Bras Endocrinol Metab 2007;51/7:1134-1142)

Keywords: Peripheral neuropathy; Foot ulceration; Peripheral arterial disease; Haluxbrachial index; Inflammatory markers 
$\mathrm{A}$ NeUropatia diabética (ND) é comumente encontrada em indivíduos com diabetes mellitus (DM) tipo 1 ou 2 (1), afetando até $50 \%$ destes (2). Há heterogeneidade de formas e de manifestações clínicas da ND na dependência do segmento do sistema nervoso acometido. A ND sensório-motora simétrica distal é a forma mais freqüente, na qual há degeneração distal retrógrada e simétrica de nervo motor ou sensitivo. O comprometimento dos nervos motores dos membros inferiores no indivíduo diabético determina hipotrofia muscular, deformidades e pontos de pressão anormais; o comprometimento dos nervos sensitivos manifesta-se por distúrbios da sensibilidade nas extremidades podendo chegar à anestesia. Cerca da metade dos indivíduos com ND sensório-motora simétrica distal é assintomática mas mantém risco de lesão insensível nos pés. A ND autonômica nas extremidades determina diminuição da função sudomotora, tornando o pé ressecado e vulnerável à ulceração. A complicação neuropática é preocupante pois, particularmente nos membros inferiores, pode causar úlceras plantares que, sem tratamento adequado, culminam com amputações. A úlcera no pé acomete $15 \%$ dos indivíduos diabéticos no decorrer de sua doença e mais de $80 \%$ das amputações são precedidas de úlcera $(3,4)$.

Nos Estados Unidos, a metade das amputações de membros inferiores ocorre em indivíduos diabéticos (5). As amputações encurtam sua vida útil e reduzem consideravelmente a qualidade de vida, impondo pesado ônus aos familiares e ao sistema de saúde. A identificação de fatores de risco e o acompanhamento desses indivíduos podem evitar este desfecho.

O risco de ulceração ou amputação está aumentado em indivíduos do sexo masculino, com DM de longa duração e mal controlado. Úlcera e amputação de membro inferior em indivíduos diabéticos também associam-se à presença de aterosclerose. Por ocasião do diagnóstico de DM, a doença arterial periférica (DAP) já está presente em $8 \%$ deles; após 10 anos, a prevalência é de $15 \%$ e, após 20 anos, de $42 \%$ (6). A claudicação intermitente, sintoma característico da DAP, pode estar ausente devido à concomitância com a ND. A pele dos pés sofre também com o comprometimento macrovascular, tornando-se fria, atrófica, com alteração de fâneros e sujeita a infecções fúngicas. A ulceração decorrente puramente da obstrução arterial pode ser diferenciada da neuropática considerando a história clínica (velocidade de instalação, presença de dor) e o exame físico (localização e aparência). Porém, sabe-se que a DAP contribui para agravar as lesões neuropáticas pré-existentes em membros inferiores, uma vez que limita o acesso de células de defesa, o fornecimento de oxigênio e nutrientes, e o necessário aporte de antibióticos para a adequada cicatrização das mesmas.

Uma parcela de indivíduos comprovadamente neuropatas não desenvolve úlceras nos pés. Seria razoável supor que os indivíduos neuropatas também com DAP seriam mais susceptíveis à ocorrência de úlceras. O exame de Doppler tem sido recomendado para diagnóstico da DAP, por meio do cálculo do índice tornozelo-braquial (ITB). A indicação de obtenção do índice hálux-braquial está estabelecida no caso de valor não-interpretável de $\operatorname{ITB}(>1,30)$.

Existem consistentes evidências de que a complicação neuropática pode ser prevenida em ambos os tipos de $\operatorname{DM}(7,8)$, justificando seu rastreamento. A perda de sensibilidade (fibras grossas) pode ser identificada pelo exame do monofilamento de $10 \mathrm{~g}$ aplicado na planta dos pés. Outro método usado para diagnóstico é o limiar de percepção vibratória (LPV), obtido por meio do bioestesiômetro, que fornece uma medida quantitativa da sensibilidade vibratória. Experiência do Reino Unido revelou que o LPV, a partir de um nível de 25 volts, esteve associado a aumento no risco de úlcera, o que não ocorreu quando percebido com 15 volts ou menos (9). Outros estudos confirmaram o ponto de corte do LPV $\geq 25$ volt como preditivo para ulceração do pé $(10,11)$. Cada aumento de 1 volt da LPV no nível basal de percepção do indivíduo associou-se a aumento de 5,6\% no risco de úlcera (12).

O conhecimento sobre a fisiopatologia da ND e fatores de risco para úlcera do pé diabético é condição fundamental para que medidas de prevenção possam ser implantadas. À medida que indivíduos diabéticos têm vivido mais, estão mais expostos a esta complicação. Estimou-se que o risco de desenvolver úlceras nos pés em alguma época das suas vidas era de $15 \%$ (5), o que pode estar subestimado.

Este estudo analisou, em uma amostra de pacientes com ND sensório-motora simétrica distal do nosso meio, a hipótese de que a concomitância com DAP predispõe à ulceração do pé. Além da associação à DAP, investigou-se se marcadores inflamatórios subclínicos também se associariam a úlceras nos pés.

\section{PACIENTES E MÉTODOS}

\section{Pacientes}

O presente estudo foi aprovado pelo Comitê de Ética da UNIFESP. Os participantes assinaram termo de consentimento livre e esclarecido antes do início do estudo. Pacientes em acompanhamento no Centro de Diabetes desta Insti- 
tuição, que compareceram a consultas de rotina no período de junho de 2004 a julho de 2005, foram convidados a participar deste estudo. Aqueles com suspeita clínica de neuropatia ou alteração do exame de monofilamento foram encaminhados a um único examinador. De 60 indivíduos encaminhados, 32 preencheram os critérios de inclusão: portadores de DM do tipo 2 em tratamento medicamentoso, com idade ao diagnóstico $\geq 40$ anos, de ambos os sexos, com alteração no exame de monofilamento segundo o Consenso Brasileiro de Complicações Crônicas da Sociedade Brasileira de Diabetes (13), que identifica neuropatia periférica (comprometimento de fibras grossas) pela presença pelo menos 2 pontos insensíveis ao exame de monofilamento de $10 \mathrm{~g}$, que apresentasse ou não história - atual ou pregressa — de úlcera não-infectada em pés. Como critérios de exclusão considerou-se história atual ou prévia de etilismo, doenças auto-imunes, doenças inflamatórias agudas, doenças carenciais e endocrinológicas com manifestações neurológicas e corticoterapia. O uso de anti-inflamatórios não-hormonais não representou critério de exclusão.

\section{Métodos}

Os indivíduos foram estratificados em 2 grupos segundo a história ou presença de úlcera nas extremidades inferiores (planta dos pés e pododáctilos). Foram incluídos no grupo "com úlcera" aqueles que apresentavam úlcera ativa ou cicatrizada, ou que tiveram alguma amputação em membro inferior decorrente de complicações da úlcera. O grupo "sem úlcera" foi constituído por aqueles indivíduos que nunca tinham apresentado úlcera em pés. Foram coletados dados de história clínica detalhada, medicamentos em uso e realizado exame físico completo, incluindo os seguintes exames complementares:

\section{Testes neurológicos}

Os testes sensoriais foram realizados com o indivíduo deitado e relaxado, em local silencioso. Para indivíduos com amputações menores (pododáctilos, pé), a avaliação neurológica restringiu-se às regiões disponíveis. No caso de amputações maiores (trans-tibial e trans-femoral), apenas o outro membro foi avaliado. Para cada modalidade de teste, inicialmente era realizado estímulo na mão do paciente para referência da sensação. Uma vez compreendida a técnica, era solicitado que fechasse os olhos e iniciava-se o teste nos membros inferiores. O monofilamento empregado foi o de $10 \mathrm{~g}$ (Semmes-Weinstein), aplicado a regiões plantares (cabeças do $1^{\circ}$, 3ำ e $5^{\circ}$ metatarsos e o $1^{\circ}$, $3^{\circ}$ e $5^{\circ}$ pododáctilos), perpendicularmente à pele, com força suficiente para causar seu encurvamento por um segundo. Considerou-se o exame do monofilamento alterado quando o indivíduo falhava em perceber 2 ou mais locais testados.

O limiar de percepção vibratória (LPV) foi obtido por meio do biotesiômerto (Biothesiometer, Biomedical Instruments, Newbury, Ohio). O aparelho era aplicado ao hálux e maléolo medial de ambos os membros; a voltagem era gradualmente aumentada (escala de 0 a 50) e o valor considerado era aquele no qual o indivíduo sentia a vibração.
Foram obtidas 3 leituras em cada local e calculada a média em cada membro. Indivíduos incapazes de sentir o estímulo receberam o valor de LPV de 51 . O maior valor da média entre os 2 membros foi utilizado para a análise. A sensibilidade dolorosa foi pesquisada com palito de madeira pontiagudo, pressionado no dorso do hálux direito e esquerdo. A pior resposta dos membros foi considerada para a análise. $\mathrm{O}$ mesmo ocorreu quando da avaliação dos reflexos aquileu e patelar em ambos os membros.

\section{Avaliação arterial de membros inferiores por Doppler}

Após repouso de 10 minutos, medidas das pressões sistólicas das artérias braquiais de ambos os membros eram aferidas com o auxílio de um transdutor de $8 \mathrm{MHz}$ (Parks Medical Eletronics, Aloha, OR, USA), adotando-se a maior medida como referência. Posteriormente, eram aferidas as pressões sistólicas das artérias pediosa dorsal e tibial de ambos os membros, novamente adotando-se a maior medida em cada membro (14). $O$ índice tornozelo-braquial (ITB) em cada membro foi calculado através da medida da maior pressão aferida no tornozelo pela maior pressão em membro superior. Além deste índice, a presença de arteriopatia obstrutiva foi investigada pela medida de pressão sistólica em hálux, utilizando-se manguito de $2,5 \mathrm{~cm}$ (peniano). Para cálculo do índice hálux-braquial adotouse a maior medida da pressão dos braços (14).

\section{Exames laboratoriais e métodos analíticos}

Os indivíduos foram submetidos às coletas de sangue em jejum para dosagens de glicemia, hemoglobina glicada (HbAlc), colesterol total e frações, triglicérides, creatinina e marcadores inflamatórios (proteína C reativa - PCR, interleucinas - IL e fator de necrose tumoral alfa - TNF- $\alpha$ ). Também foram coletadas pelo menos 2 amostras de urina de 12 horas noturnas para determinação da excreção urinária de albumina. Os participantes foram examinados por oftalmologistas para diagnóstico de retinopatia.

As análises bioquímicas foram realizadas no Laboratório Central da UNIFESP. A glicose plasmática foi determinada por kit colorimétrico, utilizando o método da glicose-oxidase e as concentrações séricas de colesterol por kits enzimáticos. Os valores de VLDL e LDL-colesterol foram determinados a partir dos valores dosados de colesterol total, HDL e triglicérides com a fórmula de Friedwald. A creatinina sérica foi medida pela reação de Jaffé. A HbAlc, determinada pelo método de cromatografia líquida de alta performance (HPLC Alc 2.2 - TOSOH; valor de referência para adultos < 7,0\%). A PCR ultrassensível foi determinada por ELISA (DSL, Webster, TX, USA); a IL-6, IL-10 e o TNF- $\alpha$ foram dosados por kit comercial (Lincoplex Cytokine Analytes, Linco Reseach, Missouri, USA).

\section{Critérios diagnósticos}

Os critérios diagnósticos para macroangiopatia foram história prévia de infarto do miocárdio, angina confirmada por alterações eletrocardiográficas compatíveis com isquemia 
miocárdica, necessidade de revascularização ou angioplastia, acidente vascular cerebral ou um valor de ITB $\leq 0,90 \mathrm{em}$ qualquer dos membros (14).

O diagnóstico das complicações microvasculares baseou-se na fundoscopia e excreção urinária de albumina. Considerou-se portador de retinopatia, indivíduo cujo exame de fundo de olho mostrava alterações compatíveis com retinopatia proliferativa ou não-proliferativa. Nefropatia diabética foi diagnosticada pela presença de albumina $>20$ $\mu \mathrm{g} / \mathrm{min}$, em pelo menos 2 amostras de urina.

Os critérios diagnósticos para dislipidemia basearamse no National Cholesterol Education Program. Desta forma, considerou-se dislipidemia, níveis séricos de colesterol total $\geq 200 \mathrm{mg} / \mathrm{dL}, \mathrm{LDL} \geq 130 \mathrm{mg} / \mathrm{dL}$, triglicérides $\geq 150$ $\mathrm{mg} / \mathrm{dL}$ ou indivíduos em uso de hipolipemiantes. Hipertensão arterial foi definida por pressão arterial sistólica ou diastólica $\geq 140$ x $90 \mathrm{mmHg}$ ou uso de anti-hipertensivos (16).

\section{Análise estatística}

Variáveis numéricas de interesse foram comparadas pelo teste $\mathrm{t}$ de Student para as amostras independentes. Quando não apresentavam distribuição normal foi utilizada a transformação logarítmica. Em se tratando de variáveis categóricas, foram empregados o teste de qui-quadrado ou exato de Fisher. Para comparar os dois índices - ITB e hálux-braquial - quanto à proporção de resultados alterados, empregou-se o teste de homogeneidade marginal. A correlação entre variáveis foi testada pelo coeficiente de Pearson. O nível de significância foi fixado em $\mathrm{p}<0,05$.

\section{RESULTADOS}

A casuística foi composta de 32 indivíduos diabéticos neuropatas, sendo 18 portadores de úlcera atual ou pregressa. Os grupos estratificados pela presença de úlcera foram semelhantes quanto à distribuição dos sexos, tendo havido predominância do sexo masculino em ambos os grupos (tabela 1). A idade variou entre 41 e 74 anos, não havendo diferença nas médias entre os grupos com e sem úlcera; também não houve diferença quanto ao tempo de diagnóstico de DM. A média da altura dos indivíduos do grupo com úlcera foi significantemente mais elevada do que aquele sem úlcera $(1,70 \pm 0,06$ vs. $1,63 \pm 0,11 \mathrm{~m} ; \mathrm{p}=0,044)$; porém, os grupos não diferiram quanto ao índice de massa corporal, circunferência abdominal, pressão arterial sistólica e diastólica. As freqüências de hipertensão arterial $(81,2 \%)$ e dislipidemia $(81,2 \%)$ do total de 32 indivíduos estudados foram altas, mas tais percentuais não foram diferentes entre os subgrupos. Cerca de $56 \%$ e $50 \%$ nos grupos com e sem úlcera, respectivamente, faziam uso de estatinas e um paciente no grupo sem úlcera usava fibrato. Similarmente, não houve diferença entre os grupos em relação ao tabagismo pregresso ou atual. A maioria dos pacientes $(81,3 \%)$ fazia uso de insulina associada ou não a antidiabéticos orais, e os demais indivíduos, apenas medicamentos orais. Considerando-se o grupo com úlcera, $12(66,7 \%)$ sofreram algum tipo de amputação em membros inferiores.

A média do LPV no maléolo medial foi significantemente maior no grupo com úlcera $(40,9 \pm$ $13,0$ vs. $30,6 \pm 12,3 \mathrm{~V} ; \mathrm{p}=0,040)$ mas apenas marginalmente significante no que se refere ao LPV no hálux $(39,4 \pm 13,8$ vs. $29,0 \pm 15,2 \mathrm{~V} ; \mathrm{p}=0,055)$ (tabela 2). Um dentre esses 32 pacientes desapareceu do serviço sem que as demais avaliações fossem realizadas.

Estratificando os reflexos e a sensibilidade dolorosa em 3 categorias - preservada, diminuída e ausente - , apenas a distribuição dos indivíduos quanto ao reflexo patelar foi significantemente diferente entre aqueles com e sem úlcera, sendo a pior resposta no grupo com úlcera $(\mathrm{p}=0,047)$. O reflexo aquileu estava ausente na maioria dos indivíduos em ambos os grupos (88,2\% no grupo com úlcera e $78,6 \%$ no grupo sem úlcera). A avaliação da sensibilidade dolorosa mostrouse mais comprometida no grupo com úlcera, embora marginalmente significante $(\mathrm{p}=0,058)$.

Ao exame de Doppler, 5 pacientes ( 3 no grupo com e 2 no grupo sem úlcera) apresentaram ITB > 1,30. Desprezando-se tais indivíduos (valores nãointerpretáveis), as médias dos ITBs direito e esquerdo foram semelhantes nos grupos com e sem úlcera (tabela 4). Do total de 31 pacientes submetidos ao ITB, em apenas 22 foi possível obter a pressão sistólica em pelo menos um dos hálux, nos quais houve adequação do manguito peniano ao diâmetro do hálux. A freqüência de alteração dos índices, em pelo menos um dos membros inferiores, foi elevada na amostra como um todo.

Considerando-se os grupos com e sem úlcera, o índice hálux-braquial foi obtido em 10 e 12 indivíduos, respectivamente. Analisando-se isoladamente cada índice em cada membro (tabela 3), o comportamento do grupo com úlcera foi sempre mais desfavorável, ou seja, com valores de ITB, índice hálux-braquial e pressão sistólica do hálux sempre mais baixos que no grupo sem úlcera, embora sem atingir significância estatística. Comparando-se as freqüências de indivíduos com o índice hálux-braquial alterado em pelo menos um dos membros, a proporção foi maior no grupo com úlcera, no qual a totalidade de indivíduos examinados apresentou alteração neste 
Tabela 1. Características gerais dos indivíduos diabéticos neuropatas, estratificados segundo a presença de úlcera em pé (atual ou pregressa). Dados expressos em número de indivíduos ou média \pm desvio-padrão.

\begin{tabular}{|c|c|c|c|c|}
\hline & $\begin{array}{c}\text { Total } \\
\mathbf{N}=\mathbf{3 2}\end{array}$ & $\begin{array}{l}\text { Sem úlcera } \\
N=14\end{array}$ & $\begin{array}{c}\text { Com úlcera } \\
\qquad=18\end{array}$ & Valor de p \\
\hline Homem / mulher (\%) & $22 / 10(68,7 / 31,3)$ & $8 / 6(57,2 / 42,8)$ & $14 / 4(77,8 / 22,2)$ & 0,267 \\
\hline Idade (anos) & $57,5 \pm 8,5$ & $58,1 \pm 8,7$ & $56,9 \pm 8,5$ & 0,699 \\
\hline Duração do diabetes (anos) & $16,5 \pm 8,9(2$ a 40$)$ & $17,9 \pm 8,2(7$ a 32$)$ & $15,4 \pm 9,5(2$ a 40$)$ & 0,434 \\
\hline Altura (m) & $1,67 \pm 0,09$ & $1,63 \pm 0,11$ & $1,70 \pm 0,06$ & 0,044 \\
\hline Índice de massa corporal $\left(\mathrm{kg} / \mathrm{m}^{2}\right)$ & $29,8 \pm 6,4$ & $28,3 \pm 5,8$ & $31,0 \pm 6,8$ & 0,262 \\
\hline Circunferência abdominal (cm) & $103,5 \pm 16,2$ & $99,3 \pm 18,6$ & $107,4 \pm 13,0$ & 0,198 \\
\hline Pressão arterial sistólica $(\mathrm{mmHg})$ & $150,0 \pm 23,6$ & $150,0 \pm 20,3$ & $150,0 \pm 26,7$ & 0,999 \\
\hline Pressão arterial diastólica $(\mathrm{mmHg})$ & $87,6 \pm 12,8$ & $88,2 \pm 11,0$ & $87,1 \pm 14,4$ & 0,816 \\
\hline Hipertensão (\%) & 81,2 & 71,4 & 88,9 & 0,365 \\
\hline Dislipidemia (\%) & 81,2 & 85,7 & 73,7 & 0,672 \\
\hline
\end{tabular}

Tabela 2. Alterações neurológicas de membros inferiores dos indivíduos diabéticos neuropatas, estratificados segundo a presença de úlcera em pé (atual ou pregressa). Dados expressos em média \pm desvio-padrão ou número de indivíduos.

\begin{tabular}{lcccc}
\hline & $\begin{array}{c}\text { Total } \\
\mathbf{N}=\mathbf{3 2}\end{array}$ & $\begin{array}{c}\text { Sem úlcera } \\
\mathbf{N}=\mathbf{1 4}\end{array}$ & $\begin{array}{c}\text { Com úlcera } \\
\mathbf{N}=\mathbf{1 8}\end{array}$ & Valor de p \\
& $35,0 \pm 15,1$ & $29,0 \pm 15,2$ & $39,4 \pm 13,8$ & 0,055 \\
LPV hálux (V) & $36,6 \pm 13,5$ & $30,6 \pm 12,3$ & $40,9 \pm 13,0$ & 0,040 \\
LPV maléolo medial (V) & $2 / 3 / 26$ & $1 / 2 / 11$ & $1 / 1 / 15$ & 0,779 \\
$\begin{array}{l}\text { Reflexo aquileu * } \\
\text { Normal / } \downarrow / \text { ausente }\end{array}$ & $13 / 4 / 14$ & $8 / 3 / 3$ & $5 / 1 / 11$ & 0,047 \\
$\begin{array}{l}\text { Reflexo patelar * } \\
\text { Normal / } \downarrow / \text { ausente }\end{array}$ & $9 / 7 / 15$ & $7 / 3 / 4$ & $2 / 4 / 11$ & 0,058 \\
$\begin{array}{l}\text { Sensibilidade dolorosa } \\
\text { Normal / } \downarrow / \text { ausente }\end{array}$ & & & & \\
\hline
\end{tabular}

LPV, limiar de percepção vibratória.

* 1 paciente desapareceu do serviço sem avaliação de reflexos e sensibilidade dolorosa.

Tabela 3. Alterações circulatórias de membros inferiores dos indivíduos neuropatas, estratificados segundo a presença de úlcera (atual ou pregressa). Dados expressos em média \pm desvio-padrão ou percentual.

\begin{tabular}{lcccc}
\hline & $\begin{array}{c}\text { Total } \\
\mathbf{N = 3 2}\end{array}$ & $\begin{array}{c}\text { Sem úlcera } \\
\mathbf{N = \mathbf { 1 4 }}\end{array}$ & $\begin{array}{c}\text { Com úlcera } \\
\mathbf{N = 1 8}\end{array}$ & Valor de p \\
Índice tornozelo-braquial direito & $0,89 \pm 0,29$ & $0,94 \pm 0,25$ & $0,85 \pm 0,32$ & 0,455 \\
Índice tornozelo-braquial esquerdo & $0,97 \pm 0,20$ & $1,03 \pm 0,13$ & $0,91 \pm 0,24$ & 0,107 \\
Índice tornozelo-braquial $\leq 0,9$ & 42,3 & 33,3 & 50,0 & 0,453 \\
em pelo menos 1 membro (\%) & & & & \\
Pressão sistólica hálux D (mmHg) & $83,9 \pm 49,9$ & $85,6 \pm 44,6$ & $80,7 \pm 61,6$ & 0,839 \\
Pressão sistólica hálux E (mmHg) & $84,3 \pm 50,7$ & $88,8 \pm 44,6$ & $78,3 \pm 60,1$ & 0,653 \\
Índice hálux-braquial D < 0,7 (\%) & 61,1 & 45,4 & 85,7 & 0,151 \\
Índice hálux-braquial E < 0,7 (\%) & 73,7 & 66,7 & 85,7 & 0,630 \\
Índice hálux-braquial < 0,7 em & 77,3 & 58,3 & 100,0 & 0,030 \\
pelo menos 1 membro (\%) & & & & \\
\hline
\end{tabular}

1 paciente desapareceu do serviço sem avaliação vascular.

O índice hálux-braquial foi obtido para 12 no grupo sem úlcera e 10 no grupo com úlcera. 
Tabela 4. Características dos exames laboratoriais e marcadores inflamatórios dos indivíduos diabéticos neuropatas, estratificados segundo a presença de úlcera em pé (atual ou pregressa). Dados expressos em média \pm desvio-padrão.

\begin{tabular}{lcccc}
\hline & Total & Sem úlcera & Com úlcera & Valor de p \\
& $\mathbf{N}=\mathbf{3 2}$ & $\mathbf{N}=\mathbf{1 4}$ & $\mathbf{N}=\mathbf{1 8}$ & 0,241 \\
Glicemia de jejum (mg/dL) & $213,0 \pm 131,0$ & $244,1 \pm 151,1$ & $184,0 \pm 106,8$ & 0,016 \\
HbA1c (\%) & $9,4 \pm 2,1$ & $10,3 \pm 2,1$ & $8,3 \pm 1,5$ & 0,038 \\
Colesterol total (mg/dL) & $220,1 \pm 52,2$ & $240,3 \pm 57,8$ & $199,9 \pm 37,9$ & 0,125 \\
LDL-c (mg/dL) & $133,6 \pm 44,5$ & $147,3 \pm 54,0$ & $147,3 \pm 54,0$ & 0,655 \\
HDL-c (mg/dL) & $45,3 \pm 13,2$ & $46,4 \pm 11,4$ & $44,1 \pm 15,1$ & 0,103 \\
Triglicérides (mg/dL) & $222,6 \pm 155,6$ & $270,6 \pm 201,2$ & $270,6 \pm 201,2$ & 0,380 \\
Creatinina (mg/dL) & $1,3 \pm 0,6$ & $1,2 \pm 0,5$ & $1,2 \pm 0,5$ & 0,883 \\
PCR (mg/dL) & $2,1 \pm 2,2$ & $2,0 \pm 1,7$ & $2,1 \pm 2,5$ & 0,678 \\
TNF- $\alpha(\mathrm{pg} / \mathrm{mL})$ & $11,2 \pm 5,8$ & $11,7 \pm 5,7$ & $10,8 \pm 6,0$ & 0,622 \\
IL-6 (pg/mL) & $10,7 \pm 12,5$ & $10,7 \pm 12,5$ & $10,7 \pm 12,5$ & 0,452 \\
IL-10 (pg/mL) & $12,6 \pm 9,8$ & $14,0 \pm 13,1$ & $11,2 \pm 4,7$ & \\
\hline PCR $/ \mathrm{proteina}$ & & &
\end{tabular}

PCR, proteína C reativa; TNF, fator de necrose tumoral; IL, interleucina.

Triglicérides e marcadores inflamatórios transformados em logaritmo para análise estatística.

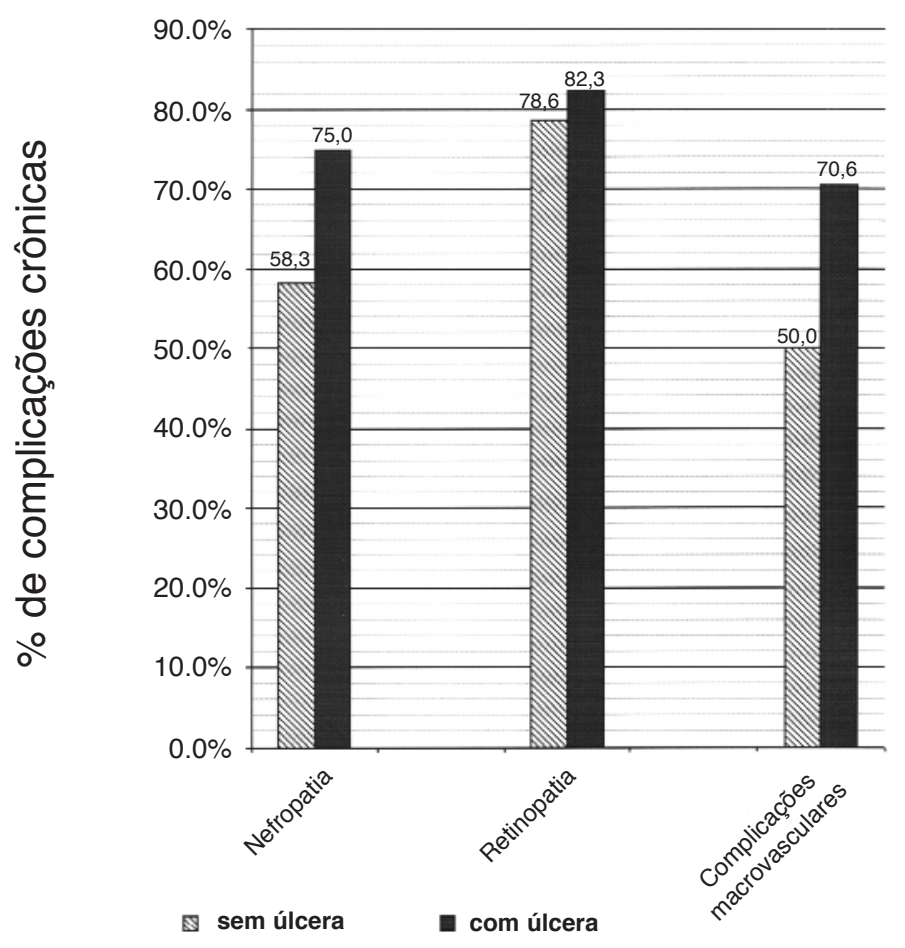

Figura 1. Freqüência dos 3 grandes grupos de complicações crônicas de indivíduos diabéticos tipo 2 estratificados segundo a presença de úlcera.

parâmetro $(\mathrm{p}=0,030)$ (tabela 3$)$. O teste de homogeneidade marginal revelou que as proporções de resultados alterados pelo ITB e índice háluxbraquial foram, de fato, discordantes $(\mathrm{p}=0,034)$.

As freqüências de nefropatia, retinopatia e macroangiopatia foram elevadas em ambos os grupos, com valores sempre maiores, embora não estatisticamente diferente, no grupo com úlcera (figura 1 ).

As concentrações plasmáticas de glicose em jejum entre os grupos não foram diferentes, embora a média de HbAlc no grupo com úlcera tenha sido estatisticamente menor $(8,3 \pm 1,5$ vs. $10,3 \pm 2,1 \%$; $\mathrm{p}=$ 
0,016) (tabela 3). Comportamento semelhante deste grupo foi observado em relação às concentrações de colesterol total $(199,9 \pm 37,9$ vs. $240,3 \pm 57,8$ $\mathrm{mg} / \mathrm{dL} ; \mathrm{p}=0,038)$. Porém, os grupos não diferiram quanto aos valores médios de LDL-c, HDL-c, triglicérides, creatinina e marcadores inflamatórios (PCR, IL-6, IL-10 e TNF- $\alpha$ ).

Não foram observadas correlações significantes entre as variáveis LPV no hálux e ITB direito $(\mathrm{r}=-0,148$, IC95\% 0,513-0,262) e ITB esquerdo $(r=-0,058$, IC95\% -0,436-0,336).

\section{DISCUSSÃO}

O presente estudo enfocou a ND sensório-motora simétrica distal, complicação de elevada prevalência e o mais importante fator de risco para úlceras plantares em indivíduos com DM $(17,18)$. A observação de que a ocorrência de ND é bem mais freqüente (50\%) (2) que a de úlcera (15\%) no DM (5) sugere que um subgrupo dos indivíduos diabéticos apresente fatores que os tornam mais predisponentes à ulceração. Fatores associados à presença ou história de úlcera foram identificados neste estudo. A hipótese de que a DAP representaria um destes fatores é sugerida, embora limitações relativas ao delineamento do estudo e tamanho da amostra não permitem afirmar tal possibilidade.

Os achados no grupo de pacientes com úlcera, comparados ao sem úlcera, de maiores valores médios de estatura e LPV e pior resposta do reflexo patelar eram esperados. Outros autores já haviam encontrado que indivíduos mais altos com nervos mais longos apresentam maior risco de desenvolver ND sensóriomotora simétrica distal (19). Na mesma linha, posteriormente, o Seattle Diabetic Foot Study encontrou, entre os mais altos, associação com a presença de úlcera (5). Alterações do LVP e reflexo patelar compatíveis com lesão sensitiva e motora, respectivamente - mais freqüentes nos ulcerados são favoráveis à participação causal desta forma de ND no aparecimento de úlcera. O LPV, sensível para diagnóstico de neuropatia leve e subclínica (20), já tinha sido apontado por Boulton e cols. (21) como preditivo de risco de ulceração. No Multicenter Study of the Incidence of Predictive Risk Factors for Diabetic Neuropathic Foot Ulceration, a incidência anual da primeira úlcera no pé em indivíduos com ND foi elevada, da ordem de 7,2\% (12). Os autores observaram que a cada aumento de 1 volt no LPV o risco da primeira úlcera no pé se elevava em 5,6\%. Incor- porando o LPV na prática clínica, pode haver considerável melhora no diagnóstico de pacientes com ND, o que minimizaria as chances de ulceração, reduzindo, conseqüentemente, os custos desta complicação (22).

O comprometimento de reflexos ocorre com a evolução da ND sensório-motora simétrica distal, sendo que em membros inferiores o aquileu é o mais precocemente acometido. Em nosso estudo, esse reflexo não serviu para diferenciar os grupos com e sem úlcera. Este fato pode ser atribuído ao tamanho da amostra, relativamente pequeno, e ao grande número de participantes com estágio avançado de ND, acompanhados em um centro de referência para atendimento de pacientes com DM. Ambos os grupos apresentaram com elevadas freqüências de resposta anormal do reflexo aquileu. Porém, a perda do reflexo patelar, que ocorre posteriormente na história natural desta complicação, foi mais freqüente no grupo com úlcera, reforçando a hipótese de apresentarem quadro neuropático de maior gravidade.

Neste estudo, comprovou-se a associação entre as complicações neuropática e microvasculares do DM previamente descrita $(1,24)$. A maioria dos indivíduos aqui avaliados apresentava retinopatia e nefropatia, com predominância no grupo com úlcera. Importantes estudos prospectivos envolvendo indivíduos com DM tipo 1 (7) e tipo 2 (8) confirmam a relação causal entre controle glicêmico precário e surgimento de complicações microvasculares e neuropáticas. Encontraram correlação direta do nível de hemoglobina glicada e o risco destas complicações (25). Contrariamente, em nosso estudo, o grupo de pacientes com úlcera apresentou menor valor de hemoglobina glicada que o grupo sem úlcera. Este achado inesperado deve provavelmente ser decorrente do fato de esses indivíduos apresentarem quadro mais grave de ND, que necessitaram acompanhamento clínico mais próximo, para cuidados com a úlcera.

Os marcadores de inflamação subclínica têm sido empregados para complementar a avaliação do risco de complicações macrovasculares. Apesar de não serem dotados da mesma capacidade preditiva de eventos que os clássicos fatores de risco cardiovascular, auxiliam na identificação de subgrupos de pacientes (26). Particularmente em indivíduos neuropatas, até onde conhecemos não está documentada associação destes com úlcera não-infectada de extremidades.

Estudos in vitro investigaram a participação de citocinas na ND, porém buscando conhecer a fisiopatologia da dor neuropática $(27,28)$. Estudos clínicos preliminares revelam concentrações de marcadores inflamatórios (IL-6 e PCR) marcadamente 
elevadas em quadros agudos de úlcera de indivíduos diabéticos $(29,30)$, havendo relação direta destes marcadores com a extensão da lesão e a gravidade da infecção. Particularmente em indivíduos neuropatas, não está documentada associação destes marcadores com risco de úlcera. O presente estudo investigou se em indivíduos portadores de úlcera (atual nãoinfectada ou pregressa) configurar-se-ia um estado inflamatório sub-clínico por meio das dosagens de PCR e interleucinas. Este achado poderia representar um elo fisiopatogênico entre as complicações neuropática e cardiovascular no indivíduo com DM. De fato, o número de indivíduos com complicações macrovasculares foi mais elevado no grupo com úlcera, embora o limitado tamanho da amostra não tenha permitido comprovar diferença estatística. Desta forma, essa hipótese não encontra suporte em nossos dados, a qual requer ser re-examinada em casuísticas maiores e estudos prospectivos.

$\mathrm{Na}$ etiologia das úlceras de pé em indivíduos com DM, atribui-se 20\% em decorrência exclusivamente da DAP, e o restante causado pela ND isolada (50\%) ou em associação à DAP (30\%). A DAP desempenha um papel importante na cicatrização de lesões e risco de amputação (4). Em nosso estudo, os parâmetros empregados para diagnóstico de comprometimento da circulação arterial nos membros inferiores foram o ITB e o índice hálux-braquial. $\mathrm{O}$ ITB, validado pela angiografia contrastada, apresenta alta sensibilidade e especificidade para detectar estenoses $\geq 50 \%$, sendo recomendado pela comunidade científica para fins diagnósticos (14). Porém, diante de artérias não-compressíveis em membros inferiores - como ocorre mais freqüentemente em pacientes diabéticos ou idosos devido à calcificação da parede arterial —, o ITB pode fornecer resultados nãointerpretáveis $(14,29)$. Isso de fato ocorreu com 5 indivíduos da nossa amostra, que apresentaram ITB > 1,30. Nestes casos tem sido sugerido o uso do índice hálux-braquial (14). Não foi propósito deste estudo analisar o valor deste último índice no caso da falta de interpretabilidade do ITB. Porém, especulando que seu emprego poderia auxiliar na identificação de DAP em artérias mais distais de membros inferiores, e na capacidade de detectar associação com a presença de úlcera, optamos por um emprego mais amplo.

Em 22 pacientes pertencentes a qualquer dos grupos, tanto o ITB quanto o índice hálux-braquial foram obtidos. Segundo esperado, não houve homogeneidade entre os resultados destes índices, favorecendo a idéia de que o último poderia estar acrescentando informação para o diagnóstico de DAP; tal hipótese necessita ser investigada em estudos desenhados para este fim. Analisando-se as freqüências de alguma evidência de DAP nesta sub-amostra de 22 pacientes, estratificados segundo a presença de úlcera, um percentual significantemente maior de indivíduos arteriopatas foi detectado no grupo com úlcera. Este achado é coerente com a hipótese do presente estudo, de que indivíduos diabéticos neuropatas com DAP poderiam apresentar risco aumentado de ulcerações em membro inferior. Porém, o delineamento deste estudo não permite estabelecer relação do tipo causa-feito.

Em conclusão, a DAP está associada com a presença de úlcera (atual ou pregressa) em membros inferiores de indivíduos diabéticos neuropatas. A pesquisa de alteração de fluxo de artérias digitais de membro inferior (no hálux) contribuiu para detectar tal associação. Por outro lado, associação de neuropatia ulcerada a marcadores inflamatórios não foi observada, não sendo possível excluí-la devido às limitações do tamanho da amostra. Estudos prospectivos deverão examinar a sensibilidade do índice hálux-braquial para identificar DAP em indivíduos diabéticos sob risco de ulceração.

\section{REFERÊNCIAS}

1. Dyck PJ, Davies JL, Wilson DM, Service FJ, Melton LJ 3rd, O'Brien PC. Risk factors for severity of diabetic polineuropathy: intensive longitudinal assessment of the Rochester Diabetic Neuropathy Study cohort. Diabetes Care 1999;22:1479-86.

2. Boulton AJM, Malik RA, Arezzo JC, Sosenko JM. Diabetic somatic neuropathies. Diabetes Care 2004;27:1458-86.

3. Boulton AJM, Vinik Al, Arezzo JC, Bril V, Feldman EL, Freeman $R$, et al. Diabetic neuropathies. Diabetes Care 2005;28:955-62.

4. Margolis DJ, Allen-Taylor L, Hoffstad O, Berlin JA. Diabetic neuropathic foot ulcer: predicting which ones will not heal. Am J Med 2003; 115:627-31.

5. Boyko EJ, Ahroni JH, Stensel V, Forsberg RC, Davignon DR, Smith DG. A prospective study of risk factors for diabetic foot ulcer. Diabetes Care 1999;22:1036-42.

6. Adler A, Boyko E, Ahroni J, Smith D. Lower extremity amputation in diabetes: The independent effects of peripheral vascular disease, sensory neuropathy and foot ulcers. Diabetes Care 1999;22:1029-35.

7. Diabetes Control and Complications Trial Research Group: The effect of intensive treatment of diabetes on the development and progression of long-term complications in insulin-dependent diabetes mellitus. N Engl J Med 1993;329:977-86.

8. U.K. Prospective Diabetes Study (UKPDS) Group: Intensive blood-glucose control with sulfonylureas or insulin compared with conventional treatment and risk of complications in patients with Type 2 diabetes (UKPDS 33). Lancet 1998;352:837-53.

9. Moss SE, Klein R, Klein B. The 14-year incidence of lowerextremity amputation in a diabetic population. Diabetes Care 1999;22:951-9.

10. Lavery LA, Armstrong DG, Wunderlich RP, Tredwell J, Boulton AJM. Diabetic foot syndrome: evaluating the prevalence and incidence of foot pathology in Mexican Americans and non-Hispanic whites from a diabetes disease management cohort. Diabetes Care 2003;26:1435-8. 
11. Armstrong DG, Lavery LA, Vela AS, Quebedeaux TL, Fleischli JG. Choosing a practical screening instrument to identify patients at risk for diabetic foot ulceration. Arch Intern Med 1998; 158:289-92.

12. Abbott CA, Vileikyte L, Williamson $\mathrm{S}$, Carrington $\mathrm{AL}$, Boulton AJM. Multicenter study of the incidence of and predictive risk factors for diabetic neuropathic foot ulceration. Diabetes Care 1998;21:1071-5.

13. Gross JL, Nehme M. Detecção e tratamento das complicações crônicas do diabetes melito: Consenso da Sociedade Brasileira de Diabetes e Conselho Brasileiro de Oftalmologia. Rev Ass Brasil 1999;45:279-84.

14. ACC/AHA 2005 Practice Guidelines for the management of patients with peripheral arterial disease (lower extremity, renal, mesenteric, and abdominal aortic): a collaborative report from the American Association for Vascular Surgery/Society for Vascular Surgery, Society for Cardiovascular Angiography and Interventions, Society for Vascular Medicine and Biology, Society of Interventional Radiology, and the ACC/AHA Task Force on Practice Guidelines (Writing Committee to Develop Guidelines for the Management of Patients With Peripheral Arterial Disease): endorsed by the American Association of Cardiovascular and Pulmonary Rehabilitation; National Heart, Lung, and Blood Institute; Society for Vascular Nursing; TransAtlantic Inter-Society Consensus; and Vascular Disease Foundation. Circulation 2006;113:463-654.

15. National Cholesterol Education Program Executive summary of the Third Report of the National Cholesterol Education Program (NCEP) Expert Panel on Detection, Evaluation and Treatment of High Blood Cholesterol in Adults. JAMA 2001;285:248-97.

16. III Consenso Brasileiro de Hipertensão Arterial. Arq Bras Endocrinol Metab 1999;43:257-86.

17. Perkins AB, Olaleye D, Zinman B, Bril V. Simple screening tests for peripheral neuropathy in the diabetes clinic. Diabetes Care $2001 ; 24: 250-6$

18. Aso $Y$, Fujiwara $Y$, Inukai $T$, Takemura $Y$. Power spectral analysis of heart rate variation in diabetic patients with neuropathic foot ulceration. Diabetes Care 1998;21:1173-6.

19. Sosenko JM, Gadia MT, Fournier AM, O'Connell MT, Aguiar MC, Skyler JS. Body stature as a risk factor for diabetics' sensory neuropathy. Am J Med 1986:80:1031-4.

20. Ziegler D, Mayer P, Wiefels K, Gries FA. Evaluation of thermal, pain and vibration sensation thresholds in newly diagnosed type 1 diabetic patients. Neurosurg Psych 1998;11:1420-4.

21. Boulton AJM, Kubrusly DB, Bowker JH, Skyler JS, Sosenko JM. Impaired vibratory perception and diabetic foot ulceration. Diabet Med 1986;3:335-7.

22. Garrow AP, Boulton AJM. Vibration perception threshold - a valuable assessment of neuronal dysfunction in people with diabetes. Diabetes Metab Res Rev 2006;22:411-9.
23. Dyck PJ, Davies JL, Wilson DM, Service FJ, Melton LJ $3^{\text {rd, }}$ O'Brien PC. Risk factors for severity of diabetic polineuropathy: intensive longitudinal assessment of the Rochester Diabetic Neuropathy Study cohort. Diabetes Care 1999;22:1479-86.

24. Savage S, Estacio RO, Jeffers B, Schrier RW. Urinary albumin excretion as a predictor of diabetic retinopathy, neuropathy, and cardiovascular disease in NIDDM. Diabetes Care 1999;22:1479-86.

25. Skyler JS. Diabetic complication. The importance of glucose control. Endocrinol Metab Clin North Am 1996;25:24354.

26. Pearson TA, Mensah GA, Alexander RW, Anderson JL, Cannon III RO, Criqui M, et al. Markers of inflammation and cardiovascular disease: application to clinical and public health practice: a statement for healthcare professionals from the Centers for Disease Control and Prevention and the American Heart Association. Circulation 2003;107:499-511.

27. Beavan S, Winteran J. Nerve growth factor (NGF) differentially regulates the chemosensitivity of adult rat cultured sensory neurons. J Neurosci 1995;15:4918-26.

28. Bogdan C, Vodovotz $Y$, Nathan C. Macrophage deactivation by interleukin-10. J Exp Med 1991;174:1549-55.

29. Rose B, Shin DI, Friese G, Othamn T, Poschen U, Herder C, et al. Sustained elevated serum concentrations of the proinflammatory cytokine IL-6 in type 2 diabetes patients with diabetic foot syndrome. Diabetologia 2005;48:A356 (abstract).

30. Lobmann R, Motzkau M, Lehnert H, Ambrosch A. Cytokine analyses in wound fluids of the diabetic foot syndrome reflects inflammation and the various healing stages. Diabetologia 2005;48:A356 (abstract).

31. Orchard TJ, Strandness Jr DE. Assessment of peripheral vascular disease in diabetes. Report and recommendations of an International Workshop - American Heart Association and American Diabetes Association. Diabetes Care 1993;16:1199-209.

Endereço para correspondência:

Sandra Roberta G. Ferreira

Departamento de Nutrição

Faculdade de Saúde Pública

Universidade de São Paulo

Av. Dr. Arnaldo 715

01246-904 São Paulo, SP

Fax: 3061-7705/3061-7701

E-mail: sandrafv@usp.br 